\title{
El área metropolitana de Valencia en el sistema global de ciudades: los efectos de la crisis económica en la red de relaciones financieras de las empresas multinacionales*
}

\author{
Julia Salom \\ Félix Fajardo \\ Universidad de Valencia. Instituto Interuniversitario de Desarrollo Local \\ Departamento de Geografía \\ julia.salom@uv.es \\ felix.fajardo@uv.es
}

\section{Resumen}

El artículo analiza el impacto que la crisis económica ha tenido sobre las redes de relaciones de propiedad-filiación de las empresas multinacionales, consideradas en la literatura científica como uno de los factores más importantes en la integración de las ciudades en la globalización. El caso de estudio es el área metropolitana de Valencia, un centro de segundo nivel en las redes económicas mundiales que ha sido duramente golpeado por los efectos de la crisis desde 2008. A partir del análisis de bases de datos de empresas multinacionales en dos fechas de referencia, 2010 y 2013, se constata que la crisis económica ha provocado un retroceso en la inserción del área metropolitana en dichas redes, un aumento de las relaciones de dependencia, y una tendencia a la reestructuración geográfica de la red en la medida en que las empresas se han reorientado hacia nuevos ámbitos de relación, especialmente Iberoamérica, como estrategia de supervivencia.

Palabras clave: ciudades globales; sistema de ciudades; empresas multinacionales; Valencia

* Este artículo se ha elaborado en el marco del proyecto de investigación «Sostenibilidad social, conectividad global y economía creativa como estrategias de desarrollo en el Área Metropolitana de Valencia» (CSO2016-74888-C4-1-R), financiado por la Agencia Estatal de Investigación (AEI) y el Fondo Europeo de Desarrollo Regional (FEDER) dentro del Programa Estatal de Investigación, Desarrollo e Innovación Orientada a los Retos de la Sociedad, en el marco del Plan Estatal de Investigación Científica y Técnica y de Innovación 2013-2016, convocatoria de 2016; y con el apoyo de una subvención concedida por el Ministerio de Educación, Cultura y Deporte dentro del Programa Nacional de Movilidad de Recursos Humanos (PRX15/00590). Félix Fajardo cuenta con una ayuda para contratos predoctorales para la formación de profesorado universitario, convocatoria 2014 (FPU14/05550), financiada por el Ministerio de Educación, Cultura y Deporte. 
Resum. L'àrea metropolitana de València dins el sistema global de ciutats: els efectes de la crisi econòmica en la xarxa de relacions financeres de les empreses multinacionals

L'article analitza l'impacte que la crisi econòmica ha tingut en les xarxes de relacions de propietat-filiació d'empreses multinacionals, considerades en la literatura científica com un dels factors més importants en la integració de les ciutats en la globalització. L'estudi de cas és l'àrea metropolitana de València, un centre de segon nivell en les xarxes globals que ha estat afectat pels efectes de la crisi des de 2008. De l'anàlisi de bases de dades d'empreses multinacionals en dues dates de referència, 2010 i 2013, s'observa que la crisi econòmica ha provocat un retrocés en la inclusió de l'àrea metropolitana en aquestes xarxes, un augment en les relacions de dependència i una tendència a la reestructuració geogràfica de la xarxa en la mesura que les empreses es van reorientar cap a nous espais de relació, especialment l'Amèrica Llatina, com a estratègia de supervivència.

Paraules clau: ciutats globals; sistema urbà; empreses multinacionals; València

Résumé. L'aire urbaine de Valencia dans le système global des villes : Les effets de la crise économique sur le réseau des liens financiers des entreprises multinationales

L'article analyse l'impact de la crise économique sur les réseaux de relations de propriétéfiliation des entreprises multinationales, considérées dans la littérature scientifique comme l'un des facteurs les plus importants pour l'intégration des villes dans la mondialisation. Il étudie l'aire métropolitaine de Valencia (Espagne), un centre de deuxième niveau dans les réseaux économiques mondiaux qui a été fortement concerné par les effets de la crise depuis 2008. À partir de l'analyse des bases de données publiques sur les sociétés multinationales à deux dates de référence, 2010 et 2013, on constate que la crise économique a entraîné un retour en arrière dans l'insertion de la zone métropolitaine dans ces réseaux, une augmentation des relations de dépendance et une tendance à la restructuration géographique du réseau dans la mesure où les entreprises ont été réorientées vers de nouveaux domaines de relation, en particulier l'Amérique latine, en tant que stratégie de survie.

Mots-clés: villes globales; système urbain; entreprises multinationales; Valencia

Abstract. The metropolitan area of Valencia in the global system of cities: The effects of the economic crisis on the network of financial links of multinational firms

This article examines how the current economic crisis has impinged on multinational firms' networks of ownership-subsidiarity relationships, considered by the scientific literature as one of the main factors that facilitate the globalization of cities. The case study is the Metropolitan Area of Valencia (Spain); a second-level centre of world economic networks that has been hard hit by the crisis since 2008. The analysis of public data sources on multinational firms for two significant dates, 2010 and 2013, shows that the economic crisis has caused a setback in the insertion of the Valencia metropolitan area in these networks, increased its dependency relationships, and reoriented the relationship network of firms toward new geographical areas, especially Latin America, as a strategy for survival.

Keywords: global cities; urban system; multinational firms; Valencia 


\section{Sumario \\ 1. Introducción 4. Conclusiones \\ 2. Fuentes y metodología Referencias bibliográficas \\ 3. Resultados}

\section{Introducción}

El presente trabajo se inscribe en la corriente que estudia la situación actual de los espacios urbanos en el marco de la globalización, y que remarca la importancia de las relaciones extralocales en el futuro de los territorios. Este punto de vista, que parte del hecho de que un número creciente de ciudades interacciona con núcleos urbanos ubicados en espacios distantes, adopta una perspectiva relacional que se centra en el análisis de las relaciones interurbanas en un contexto global y estudia el «espacio de los flujos» (Castells, 1996), basado en las conexiones, como perspectiva complementaria y necesaria del estudio del «espacio de los lugares» de Christaller, articulado en función de la proximidad geográfica. Las aportaciones más conocidas de este enfoque son los estudios de las ciudades globales o mundiales (Hall, 1966; Friedmann y Wolff, 1982; Sassen, 1991), que concluyen en la determinación de una jerarquía de ciudades mundiales encabezada por los lugares estratégicos en la nueva división internacional del trabajo, habitualmente identificados con los centros urbanos que concentran las sedes centrales de las empresas multinacionales y las actividades de servicios empresariales avanzados (Cohen, 1981; Taylor, 2001).

Sin embargo, en los últimos años ha habido distintas aportaciones críticas que han subrayado algunos de los aspectos considerados excesivamente simplistas de estos planteamientos. Así, se ha remarcado la existencia de distintos procesos de globalización, que implican a diferentes sectores económicos y no solo a los anteriormente mencionados, y que cuestionan la dicotomía entre los sectores de la moderna economía, relacionados con la globalización, y los sectores de la antigua economía, relacionados con los recursos naturales e industrias transformadoras, que permanecerían al margen de este proceso (Alderson y Beckfield, 2004; Gavinha, 2008). Por otra parte, se ha insistido en la necesidad de considerar no solo las ciudades cabecera de la jerarquía, sino también las ciudades «en globalización", es decir, las situadas en distintas fases del proceso (Krätke, 2014; De Mattos, 2010). Otras aportaciones han matizado el significado de los conceptos implícitos de «dominación» y «control», subrayando el hecho de que no se trata de juegos de suma cero, en los que las ciudades «subordinadas» puedan perder respecto a las ciudades cabecera de la jerarquía, sino de que el «poder» de las ciudades globales consiste más bien en la posibilidad de crear y mantener conexiones, un poder que no restringe necesariamente las posibilidades económicas de las ciudades «subordinadas» (Allen, 2010; Smith, 2014). Finalmente, desde un punto de vista más general, también se ha criticado el sesgo excesivamente económico del análisis, que tiende a 
subestimar el papel de las redes culturales, sociales y políticas que vinculan las ciudades a escala internacional (Pflieger y Rozenblat, 2010).

Más allá de estos debates, las críticas han provocado un cambio de perspectiva en el estudio de las economías y sistemas urbanos, que adopta ahora un enfoque relacional, de acuerdo con el cual la ciudad se considera el lugar en donde se ubica y articula una gran diversidad de nodos de redes industriales, económicas, sociales y culturales (Castells, 2010; Rozenblat, 2010). Todas las metrópolis internacionales, sean o no ciudades «globales», pueden considerarse una yuxtaposición de entidades interrelacionadas que son tanto globales como locales, en las que varía el tipo de glocalización en función de la escala, topología, estructura, funciones y evolución temporal de las redes en que se insertan (Pflieger y Rozenblat, 2010).

Para analizar la posición de las ciudades en la jerarquía global se utilizan frecuentemente, por su mayor disponibilidad, datos sobre la dotación urbana de funciones relacionadas con su proyección internacional: actividades económicas, especialmente terciarias y cuaternarias; riqueza generada, accesibilidad a redes internacionales, ferias y salones, número de hoteles, número de estudiantes, número de filiales de empresas transnacionales, etc. (Halbert et al., 2012). Sin embargo, se considera preferible utilizar datos relacionales, medidos a partir de flujos (de personas, bienes, información, conocimiento o dinero) entre los distintos núcleos urbanos (Derudder y Witlox, 2005). En esta línea, en algunos casos se han utilizado como información de base las redes de transporte interurbano e internacional, especialmente las conexiones aéreas o los flujos de pasajeros por avión (Derudder y Witlox, 2008; Córdoba Ordóñez y Gago García, 2010, etc.). En otros casos, como ocurre en el presente artículo, el importante papel de las funciones terciarias y cuaternarias en la comunicación de información, ideas, conocimiento y formación ha llevado a centrar el análisis en los flujos financieros y de servicios avanzados, con especial atención a las redes de las empresas multinacionales. Las redes de empresas multinacionales se consideran, independientemente de su sector de actividad, uno de los elementos principales de la red de relaciones que conecta el sistema urbano global (Alderson y Beckfield, 2004; Wall y Van der Knaap, 2011). La red de relaciones financieras entre empresas es vista como un sistema de canales para los flujos de capital entre ciudades, diferenciando entre las relaciones de subsidiariedad (flujos de capital entrantes), que caracterizan la ciudad como destino de inversión, y las relaciones de propiedad (flujos de salida de capital), que caracterizan la ciudad como fuente y centro de control de la inversión realizada. El primer tipo de relación puede interpretarse como una medida del potencial de atracción del área urbana o «prestigio relacional», mientras que el segundo se considera una medida de la capacidad de control o "poder relacional». Así, la estructura de relaciones de propiedadfiliación dibuja un «sistema de canales» a través de los cuales pueden circular flujos de información, conocimiento y capital que condicionan el futuro de la economía metropolitana (Rozenblat, 2004; Bellwald y Rozenblat, 2012; Luthi et al., 2013; Krätke, 2014). 
Desde esta perspectiva relacional, la posición en la jerarquía urbana estaría definida por el papel que desempeñan las ciudades en la red, de forma que los centros que están en la cúspide ocuparían posiciones estratégicas de intermediación a escala mundial, mientras que en un segundo nivel se encontrarían los lugares que, aunque no tienen el mismo tamaño ni el alcance espacial de los primeros, tienen la función de conectar países y territorios con el resto de la economía mundial. Esta última sería la función ejercida por el área metropolitana de Valencia (AMV) en el sistema mundial de ciudades, de acuerdo tanto con su dotación funcional como con su posición en las redes globales. Desde el primer punto de vista, en los estudios sobre el sistema de ciudades europeo (Brunet, 1989; Cattan et al., 1994; Halbert, Cicille y Rozenblat, 2012), Valencia aparece situada en un segundo nivel de la jerarquía, por detrás de Madrid y Barcelona, como ciudad que detenta algunas funciones internacionales (comercio, industria), pero con un cierto déficit de servicios avanzados y un nivel funcional menor de lo que le correspondería por su tamaño demográfico (Halbert, Cicille y Rozenblat, 2012). Desde la segunda perspectiva, y más concretamente en el ámbito de las relaciones financieras de las empresas multinacionales, aparece definida como un nodo intermedio/intermediario en el ámbito nacional, con un doble papel: por un lado, acoge empresas filiales detentadas por sedes externas de forma directa o indirecta (a través de empresas relays o intermediarias localizadas en el área urbana); en segundo lugar, es ámbito de localización de filiales-sedes que, siendo filiales de otras empresas, funcionan a su vez como sedes de firmas localizadas fuera del área metropolitana. Su ámbito territorial de relación abarca también ciudades europeas e iberoamericanas, pero se concentra especialmente en el litoral mediterráneo y este de España (Salom, 2014).

En el periodo previo a la crisis económica, existían en el área dos redes de relaciones paralelas y diferenciadas: una red casi exclusivamente nacional, dominada por empresas del sector de la construcción con un bajo nivel tecnológico; y una red internacional, constituida por manufacturas con un nivel tecnológico superior a la media regional y por empresas de servicios, en su mayoría intensivos en conocimiento. En el primer caso, el $40 \%$ de las relaciones de propiedad y el $58 \%$ de las relaciones de subsidiariedad implicaban a empresas constructoras o vinculadas al sector de la construcción, mientras que el resto pertenecía mayoritariamente al sector servicios, especialmente servicios no intensivos en conocimiento. En este caso, las manufacturas suponían solo entre el 7 y el $9 \%$ de las relaciones financieras interempresariales. Por el contrario, en las relaciones internacionales, las empresas manufactureras y los servicios intensivos en conocimiento protagonizaban la mayor parte de las relaciones de propiedad-filiación, mientras que las empresas del sector de la construcción y de la producción y distribución de agua, gas y electricidad eran prácticamente inexistentes. El sector manufacturero tenía una mayor presencia en el caso de las sedes valencianas con filiales en otros países (en torno al $60 \%$ de las relaciones vinculan empresas de este sector, de ellas más de un tercio de nivel tecnológico medio-alto o alto); mientras que en el caso inverso (sedes en 
el extranjero con filiales en el área urbana de Valencia), abundaban algo más las empresas del sector servicios (casi un $60 \%$ del total), intensivos en conocimiento en el caso de las sedes. Finalmente, las relaciones financieras interempresariales a escala local (sedes y filiales en el área metropolitana) se realizaban entre empresas principalmente del sector de la producción y distribución de agua, gas y electricidad (63\% de las sedes; $51 \%$ de las filiales), y, de manera secundaria, de servicios poco intensivos en conocimiento (Salom, 2014).

Por tanto, el área metropolitana de Valencia ejercía en el periodo previo a la crisis una función significativa en ese segundo nivel de ciudades cuya función principal es conectar ciudades y territorios; en este caso ejercida principalmente a escala nacional, aunque también con conexiones internacionales de cierto interés. Sin embargo, la crisis económica la golpea con fuerza y provoca un retroceso económico superior a la media española; entre 2008 y 2014, desaparece un $12,4 \%$ de las empresas de la provincia, lo que supone un retroceso superior al nacional $(-8,9 \%)$, mientras que el PIB regional se reduce en un 9,8 \% (6 \% en España) y la tasa de paro provincial pasa del 9 al 27,5\% (de 9,5 a 25,9 en España). En este marco, el objetivo de este artículo es mostrar en qué medida este impacto ha afectado la red de relaciones interempresariales, y averiguar si la estructura territorial y sectorial de la red se ha visto transformada. En particular, nos interesa verificar si las empresas articuladas por la red internacional han tenido una evolución mejor que la media, y si esta red puede constituirse en un factor de resiliencia regional.

\section{Fuentes y metodología}

En la presente investigación se ha aplicado la metodología desarrollada por Rozenblat (Rozenblat, 2004, 2010; Rozenblat y Pumain, 2007) para el análisis de las redes de empresas multinacionales, que adopta una concepción jerárquica de la empresa multinacional en la que los enlaces se caracterizan por relaciones que van desde la sede hacia sus filiales. Las relaciones financieras entre empresas se localizan en los dos extremos, origen y destino; y se agregan posteriormente por ciudades, distinguiendo entre las relaciones «entrantes» al área urbana (de filiación: empresas ubicadas en la ciudad que son participadas por otra empresa ubicada en el exterior), las relaciones «salientes» (de propiedad: empresas urbanas que detentan en parte o totalmente la propiedad de otra empresa localizada fuera del área urbana) y las relaciones locales (la empresa sede y la empresa filial se localizan en el interior del área urbana). La medida de centralidad urbana viene dada por la suma de las conexiones de las empresas ubicadas en cada ciudad (nodos), y la conectividad entre las ciudades viene medida por la suma de las relaciones entre las empresas ubicadas en cada ciudad (arcos).

La fuente utilizada es la base de datos ORBIS, publicada por el Bureau Van Dijk (BvD) en dos fechas: 2010, que debido a las características del proceso de actualización de la fuente recoge información en torno a 2008; y 2013, con datos de 2011 aproximadamente. Esta base de datos proporciona información de las 3.000 primeras empresas mundiales por su cifra de negocio, lo que 
Figura 1. Área de estudio

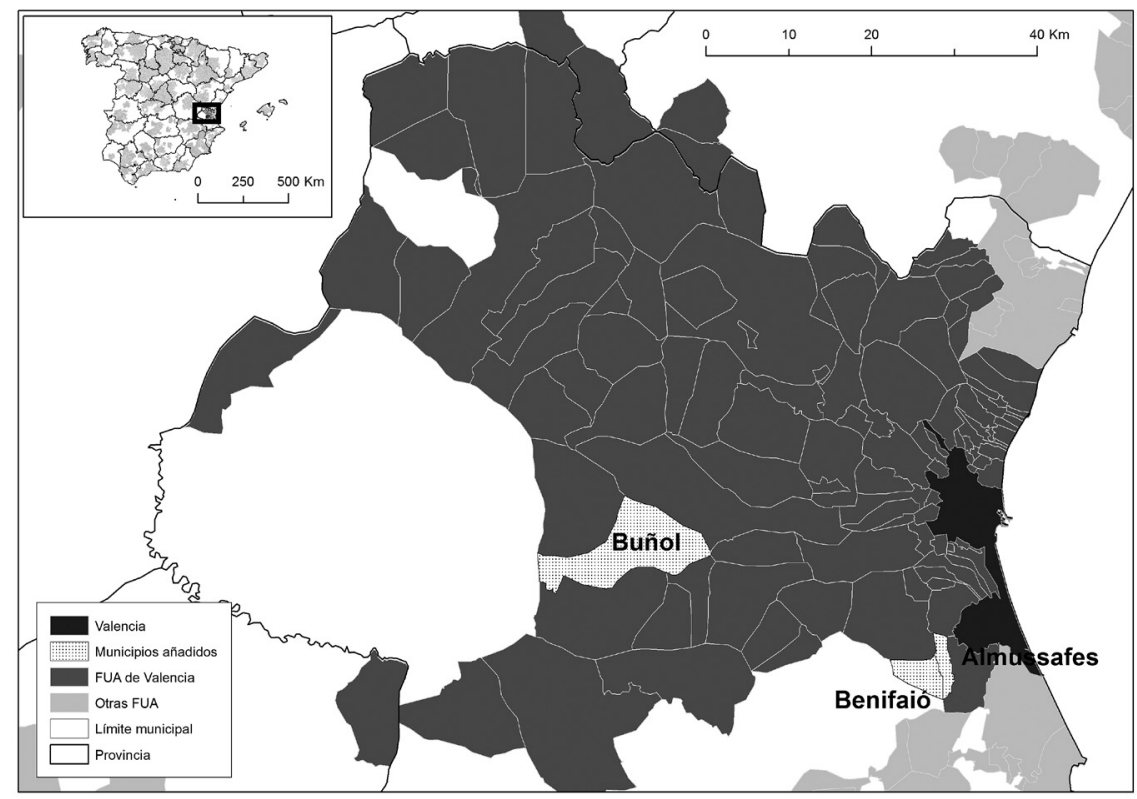

Fuente: ESPON FOCI (2011) y elaboración propia.

supone información sobre unas 800.000 filiales $^{1}$ repartidas por todo el mundo, relacionadas por aproximadamente un millón de enlaces. Esta base ha sido completada por el grupo de investigación CITADYNE de la Universidad de Lausana con la localización y delimitación de las ciudades mundiales².

En nuestro caso, hemos trabajado con una parte de esta base de datos, compuesta por las relaciones de propiedad-filiación de las empresas localizadas en Valencia, las empresas de fuera de Valencia participadas por empresas valencianas, y las empresas externas con filiales en Valencia, un total de 1.925

1. Aunque en términos estrictos una empresa puede considerarse filial de otra solo cuando la sede supere el $50 \%$ de la propiedad, en este análisis se ha optado, al igual que se ha hecho en los estudios mencionados anteriormente, por representar cualquier relación de propiedad como de filiación, ya que la fuente utilizada no proporciona información completa sobre el porcentaje de control de capital detentado por la empresa sede sobre la subsidiaria.

2. La delimitación de las áreas funcionales urbanas (FUA) se ha basado, en el caso de las ciudades europeas, en la utilizada en los estudios ESPON FOCI (2011) y DATAR (2012). Esta delimitación, comparable para todas las ciudades de la Europa de los 27 (más Suiza y Austria), ha sido elaborada por Didier Peeters (IGEAT, ULB Bruxelles) sobre la base de las migraciones alternantes, agregando aquellas unidades administrativas que envían al menos un $20 \%$ de su población activa a la aglomeración urbana, delimitada su vez a partir de la continuidad del espacio construido). Delimitaciones similares se han utilizado en el caso de las ciudades de América del Norte, China, India y Rusia. Para el resto del mundo, se han realizado agregaciones específicas de las aglomeraciones urbanas. 
relaciones de propiedad-filiación. La aplicación del análisis a una escala más detallada de lo habitual nos ha permitido realizar un proceso de depuración y control de la base de datos consistente en el contraste de la información original con las bases de datos españolas SABI (Sistema de Balances Ibéricos Bureau Van Dijck) y E-Informa (D\&B) (<http://www.einforma.com/cif-empresas $>$ ), a partir de su nombre y código de identificación fiscal. La delimitación territorial del área funcional de Valencia es la utilizada en los estudios ESPON FOCI ${ }^{3}$ y rectificada para incluir los municipios de Almussafes, Benifaió y Buñol, que no aparecen incluidos en dicha delimitación pero que, según diferentes estudios, forman indudablemente parte del área desde un punto de vista funcional (Feria, 2008 y Salom, 2011). Esta área, representada en la figura 1, está compuesta por 107 municipios que sumaban 1.793.363 habitantes en 2014 (70\% de la población provincial).

\section{Resultados}

\subsection{La evolución de la posición del $A M V$ en la red global de ciudades}

El análisis de la evolución de las relaciones financieras de empresas multinacionales entre 2010 y 2013 (tabla 1) evidencia una importante reducción en el número de vinculaciones entre ambas fechas que alcanza a casi una cuarta parte de los enlaces preexistentes $(-24,4 \%)$ y es muy superior a la experimentada por el conjunto de España $(-7,8 \%)$. Una observación más detallada nos indica que, aunque el retroceso es superior a la media española a todas las escalas, la mayor reducción se ha producido a escala local, en donde el número de relaciones de propiedad-filiación disminuye en un $64 \%$, valor que se sitúa muy por encima de la media española, cuyo retroceso es también significativo (-19\%).

Estas diferencias podrían ser el resultado de tres procesos. En primer lugar, del cierre de empresas a consecuencia de la crisis económica, que ha golpeado con fuerza, y en mayor medida que en el resto de España, a la economía valenciana. En segundo lugar, de una reestructuración financiera que hubiera provocado la reducción del número de relaciones financieras por empresa a través de procesos de desinversión o readquisición de activos. En tercer lugar, de una retirada de la participación financiera por parte de las empresas multinacionales que hubiera tenido como consecuencia su desvinculación de la red de empresas locales y, por tanto, la desaparición de estas últimas de la base de datos que, no lo olvidemos, está constituida "de arriba abajo», es decir, por las relaciones financieras en cascada a partir de las principales empresas multinacionales mundiales.

En una primera aproximación, la primera de las hipótesis parece la más acertada, ya que, como muestra la tabla 2 , el retroceso del número de empresas en la provincia de Valencia a lo largo del periodo de crisis ha sido superior a la media española en todas las categorías de tamaño, salvo en el caso de las empresas de quinientos y más trabajadores. Por el contrario, el número de relaciones por

3. Ver nota 2. 
Tabla 1. Evolución del número de relaciones financieras interempresariales según tipo de relación

\begin{tabular}{|c|c|c|c|c|c|c|}
\hline \multirow[b]{2}{*}{$\begin{array}{l}\text { Número de relaciones financieras } \\
\text { interempresariales en el AMV }\end{array}$} & \multicolumn{2}{|c|}{ Orbis 2010} & \multicolumn{2}{|c|}{ Orbis 2013} & \multicolumn{2}{|c|}{$\begin{array}{c}\text { Evolución } 2010-2013 \\
\text { (\% incremento) }\end{array}$} \\
\hline & Número & $\%$ & Número & $\%$ & $\begin{array}{c}\text { FUA } \\
\text { Valencia }\end{array}$ & España \\
\hline De subsidiariedad & 1.254 & 65,1 & 912 & 73,1 & $-27,3$ & $-12,3$ \\
\hline $\begin{array}{l}\text { Locales (filiales localizadas en Valencia } \\
\text { cuya sede se localiza en Valencia) }\end{array}$ & 437 & 29,4 & 155 & 13,8 & $-64,5$ & $-19,1$ \\
\hline $\begin{array}{l}\text { Externas (filiales localizadas en Valencia } \\
\text { cuya sede se localiza fuera del área } \\
\text { urbana) }\end{array}$ & 817 & 54,9 & 750 & 66,7 & $-8,2$ & 11,2 \\
\hline $\begin{array}{l}\text { Sin datos (filiales localizadas en Valencia } \\
\text { de las que se desconoce la localización } \\
\text { geográfica de la sede) }\end{array}$ & 0 & 0,0 & 7 & 0,6 & - & - \\
\hline De propiedad & 671 & 34,9 & 335 & 26,9 & $-50,1$ & $-13,2$ \\
\hline $\begin{array}{l}\text { Locales (sedes localizadas en Valencia } \\
\text { con filiales valencianas) }\end{array}$ & 437 & 29,4 & 155 & 13,8 & $-64,5$ & $-19,1$ \\
\hline $\begin{array}{l}\text { Externas (sedes localizadas en Valencia } \\
\text { con una filial fuera del área urbana) }\end{array}$ & 234 & 15,7 & 180 & 16,0 & $-23,1$ & 1,3 \\
\hline $\begin{array}{l}\text { Sin datos (sedes localizadas en Valencia } \\
\text { de las que se desconoce la localización } \\
\text { geográfica de la filial) }\end{array}$ & 0 & 0,0 & 33 & 2,9 & - & - \\
\hline Total & 1.488 & 100,0 & 1.125 & 100,0 & $-24,4$ & $-7,8$ \\
\hline
\end{tabular}

Fuente: Orbis $(2010,2013)$ BvD, Univ. de Lausana y elaboración propia.

Tabla 2. Evolución del número de empresas 2007-2013.

\begin{tabular}{lcc}
\hline Tamaño de la empresa & \multicolumn{2}{c}{ Evolución 2007-2013 (\%) } \\
\cline { 2 - 3 } (número de trabajadores) & Provincia de Valencia & España \\
\hline Sin asalariados & $-2,23$ & $-1,44$ \\
De 1 a 9 & $-10,42$ & $-7,20$ \\
De 10 a 99 & $-40,29$ & $-32,31$ \\
De 100 a 199 & $-26,52$ & $-21,21$ \\
De 200 a 499 & $-19,35$ & $-18,21$ \\
500 y más & $-8,43$ & $-12,18$ \\
\hline Total & $-8,22$ & $-5,70$ \\
\hline
\end{tabular}

Fuente: DIRCE y elaboración propia.

empresa se ha mantenido prácticamente igual en cifras globales, e incluso se ha incrementado ligeramente, ya que ha pasado entre 2010 y 2013 de 1,55 a 1,63. Sin embargo, respecto a este último punto, existen notables diferencias en función del tipo y escala de relación que se considere, que comentaremos más adelante.

Un segundo aspecto relevante del cambio producido entre 2010 y 2013 es el aumento de la dependencia externa de las empresas valencianas en su con- 
texto internacional. Las relaciones financieras con empresas multinacionales del AMV ya eran antes de la crisis más de dependencia que de control, ya que el número de relaciones de subsidiariedad superaba en mucho al de relaciones de propiedad; es decir, que el número de empresas valencianas controladas, total o parcialmente, por capital financiero externo superaba al de las empresas valencianas que tenían participación en empresas ubicadas en el exterior. Esta caracterización de la red como centro financiero dependiente se ha incrementado notablemente entre las dos fechas estudiadas. Así, la ratio entre relaciones de subsidiariedad y relaciones de propiedad ha pasado de ser de casi el doble de las primeras respecto a las segundas $(1,9)$ en 2010, a casi el triple en 2013 $(2,7)$. La tabla 1 muestra que el número de enlaces de propiedad (es decir, participaciones de empresas valencianas en empresas ubicadas fuera del área urbana) se ha reducido a la mitad, mientras que el número de enlaces de subsidiariedad (participaciones en empresas valencianas de sedes ubicadas fuera del área urbana) ha experimentado un retroceso de solo un $25 \%$. Aunque en ambos casos la pérdida supera la media española, el descenso en el caso de las relaciones de subsidiariedad es solo el doble del nacional, mientras que el retroceso de las relaciones de propiedad es más de cuatro veces superior a la media. Si nos centramos en los enlaces no locales, nos encontramos también un muy ligero descenso en las relaciones de filiación con empresas ubicadas fuera del área metropolitana $(-8 \%)$, frente al importante retroceso de las relaciones de propiedad externas, que alcanza un $-23 \%$, tendencia que no es posible observar en el conjunto de España.

Como veremos a continuación, este aumento de la dependencia está relacionado tanto con la evolución diferencial del número de empresas en función de su papel en la articulación de las redes financieras, como con la existencia de una reestructuración financiera concentrada en sectores económicos concretos.

\subsection{La evolución de las empresas según su posición en la red}

Para analizar este punto, es necesario partir de una clasificación de las empresas implicadas en la red valenciana de relaciones financieras interempresariales desde un punto de vista relacional, y diferenciar entre las que son cabecera de un grupo empresarial autónomo (solo el Grupo Roig, del que forma parte Mercadona), las que funcionan como un nodo en la red y que son a la vez filiales de empresas externas al área y sedes para empresas locales o externas, y las filiales absolutas. Dentro de este último grupo hay que distinguir entre las que son subsidiarias de sedes ubicadas fuera del AMV y las que lo son de otras empresas locales (ver figura 2). La hipótesis que se plantea es que la posición en la red y, en consecuencia, el tipo de relaciones que mantiene con el resto del sistema han sido un factor de diferenciación en la pervivencia de la integración de las empresas en la red global de relaciones financieras.

A este respecto, un análisis comparativo permite apreciar que la reducción ha afectado especialmente al grupo de empresas ubicadas en el AMV que son 
Figura 2. Tipología de empresas en función de su posición en la red de propiedad-filiación

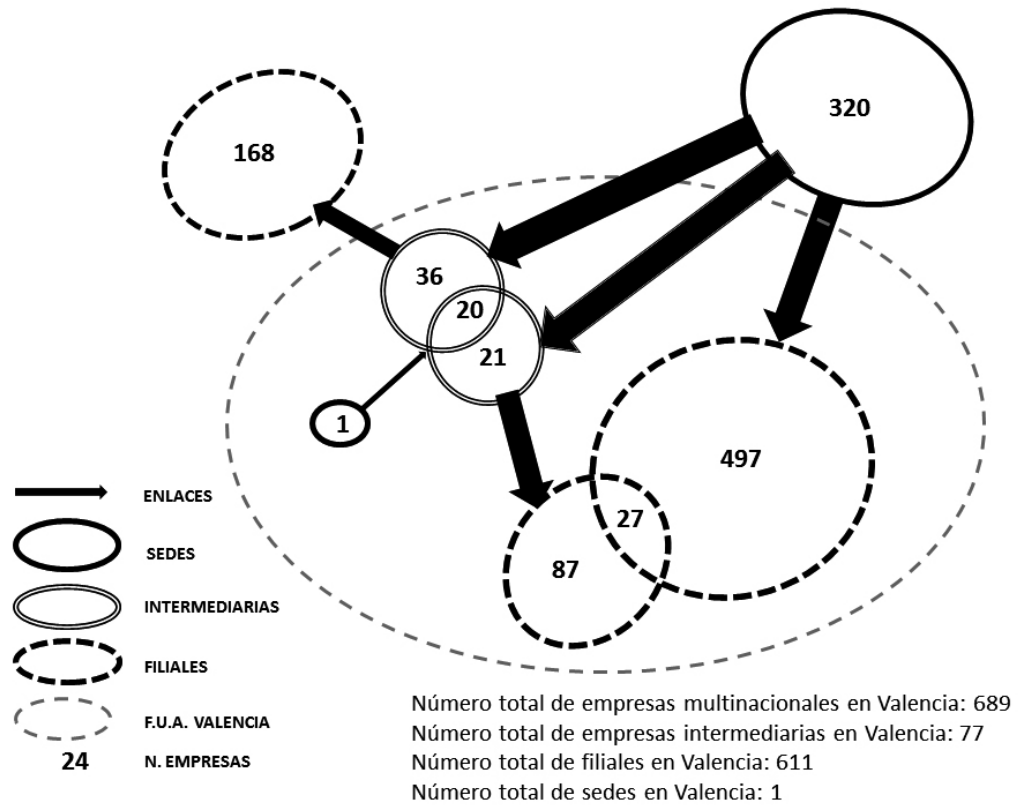

Fuente: BvD, Univ. de Lausana y elaboración propia.

filiales de otras empresas de ámbito local (-75\%). Por el contrario, la reducción del número de empresas intermediarias, que actúan como filiales-sede, es sustancialmente menor (-31\%). La mejor evolución es la seguida por las empresas que mantienen relaciones financieras con el exterior del área, sea como filiales, sea como sedes, cuyo número permanece prácticamente invariado entre ambas fechas (tabla 3).

Además de la reducción del número de empresas, en el ámbito local se ha producido una importante reestructuración de las relaciones, que ha dado como resultado una simplificación de la red, de forma que el número de relaciones financieras por empresa se ha reducido notablemente, sobre todo en el caso de las relaciones de control ${ }^{4}$ (tabla 4). Así, el número de enlaces de subsidiariedad por empresa pasa de 6,6 a 3,8 entre las dos fechas estudiadas. Esto significa que cada empresa-sede ubicada en el AMV, que tenía participaciones por término medio en algo más de seis empresas en 2010, ha pasado a tener participación en menos de cuatro empresas en 2013. Esto está relacionado con la retracción del capital financiero derivada de la crisis de los sectores energético y de la construcción, como comentaremos en el siguiente apartado.

4. En cada caso se considera el número de empresas del área de Valencia implicadas en ese tipo de relación. 
Tabla 3. Evolución del número de empresas en función de su posición en la red, 2010-2013

\begin{tabular}{lrrc}
\hline Tipo de empresa & 2010 & 2013 & Evolución 2010-2013 (\%) \\
\hline Empresas externas al área & 599 & 488 & $-18,5$ \\
Propietarias de filiales en el AMV & 375 & 320 & $-14,7$ \\
Filiales de empresas del AMV & 224 & 168 & $-25,0$ \\
\hline Empresas del AMV & 962 & 689 & $-28,4$ \\
Sedes & 0 & 1 & - \\
Intermediarias & 112 & 77 & $-31,3$ \\
$\quad$ Con filiales en el exterior & 36 & 36 & 0,0 \\
Con filiales en el AMV & 33 & 21 & $-36,4$ \\
Con filiales en el exterior y en el AMV & 33 & 20 & $-39,4$ \\
Filiales & 860 & 611 & $-29,0$ \\
$\quad$ Con sede en el exterior & 500 & 497 & $-0,6$ \\
Con sede en el AMV & 350 & 87 & $-75,1$ \\
Con sede en el exterior y en el AMV & 10 & 27 & 170,0 \\
\hline
\end{tabular}

Fuente: Orbis, Univ. de Lausana $(2010,2013)$, y elaboración propia.

Tabla 4. Evolución del número de relaciones financieras interempresariales por empresa según el tipo de relación, 2010-2013.

\begin{tabular}{lcc}
\hline & \multicolumn{2}{c}{ N. ${ }^{\circ}$ de relaciones por empresa } \\
\cline { 2 - 3 } \multicolumn{1}{c}{ Tipo de relación } & $\mathbf{2 0 1 0}$ & $\mathbf{2 0 1 3}$ \\
\hline De subsidiariedad & 1,46 & $\mathbf{1 , 4 9}$ \\
Locales de subsidiariedad (filiales en AMV con sede en AMV) & 1,21 & 1,36 \\
De subsidiariedad externa (filial en AMV con sede externa) & 1,33 & 1,31 \\
De propiedad & 6,58 & 4,29 \\
Locales de control (sedes en AMV con filial en AMV) & 6,62 & 3,78 \\
De propiedad externa (sedes en AMV con filial externa) & 3,39 & 3,16 \\
\hline Total & 1,55 & 1,63 \\
\hline
\end{tabular}

Fuente: Orbis $(2010,2013)$.

Por tanto, como síntesis de este punto, podemos decir que las empresas valencianas que se han mantenido en la red global son principalmente aquellas que tenían conexiones financieras externas al área, mientras que el retroceso más importante se ha dado entre las empresas intermediarias con filiales en el AMV. En este último caso, se ha producido una importante reestructuración y simplificación de la red de relaciones que, como veremos a continuación, tiene que ver con el estallido de dos burbujas: la burbuja de las energías renovables y la burbuja del sector de la construcción.

\subsection{Evolución por sectores económicos}

La distinción más relevante en el ámbito de las redes financieras globales es la que clasifica los sectores de actividad en función de su nivel tecnológico en 
Tabla 5. Evolución del número de empresas implicadas en relaciones multinacionales en el AMV por sectores económicos

\begin{tabular}{|c|c|c|c|c|c|}
\hline \multirow[b]{2}{*}{ Sector } & \multicolumn{2}{|c|}{$\begin{array}{c}\text { Empresas } \\
2010\end{array}$} & \multicolumn{2}{|c|}{$\begin{array}{c}\text { Empresas } \\
2013\end{array}$} & \multirow{2}{*}{$\begin{array}{c}\text { Evolución } \\
2010-2013(\%)\end{array}$} \\
\hline & Número & $\%$ & Número & $\%$ & \\
\hline Agricultura e industrias extractivas & 6 & 0,6 & 3 & 0,4 & $-100,0$ \\
\hline Agua, gas y electricidad & 237 & 24,6 & 51 & 7,4 & $-364,7$ \\
\hline Manufacturas & 76 & 7,9 & 51 & 7,4 & $-49,0$ \\
\hline De nivel tecnológico alto y medio-alto & 28 & 2,9 & 24 & 32,2 & $-16,7$ \\
\hline De nivel tecnológico bajo y medio-bajo & 48 & 5,0 & 27 & 3,9 & $-77,8$ \\
\hline Servicios & 565 & 58,7 & 516 & 74,6 & $-9,5$ \\
\hline Intensivos en conocimiento & 144 & 15,0 & 87 & 12,6 & $-65,5$ \\
\hline Menos intensivos en conocimiento salvo 9499 & 128 & 13,3 & 100 & 14,5 & $-28,0$ \\
\hline Otras actividades asociativas n.c.a. (9499) & 293 & 30,5 & 329 & 47,5 & 10,9 \\
\hline Construcción & 78 & 8,1 & 45 & 6,5 & $-73,3$ \\
\hline Sin clasificar & & & 26 & 3,8 & - \\
\hline Total & 962 & 100 & 692 & 100 & $-39,02$ \\
\hline
\end{tabular}

Fuente: Orbis, Univ. de Lausana $(2010,2013)$, y elaboración propia.

el caso de las manufacturas, y según su nivel de intensidad en conocimiento en el caso de los servicios. Las redes de ambos grupos muestran importantes diferencias de comportamiento a escala global (Rozenblat et al., 2016). En este trabajo, hemos considerado necesario además clasificar aparte la categoría «Otras actividades asociativas», ya que esta agrupa las uniones temporales de empresas (UTE), cuya actividad real en España está vinculada principalmente al sector de la construcción y solo muy escasamente al sector servicios.

En la evolución entre 2010 y 2013 (tabla 5) destaca, en primer lugar, el fuerte retroceso del sector de agua, gas y electricidad, que en nuestro caso se relaciona estrechamente con el sector de la producción y distribución de energía solar. Este sector se ha visto afectado por un importante cambio normativo que ha provocado la crisis de numerosas empresas. Los importantes subsidios concedidos a las energías renovables a partir del RD 661/2007 favorecieron la creación de empresas intermediarias que ayudaban a familias y pequeños inversores a hacer las instalaciones y registrarse como productores de energía. La nueva legislación desarrollada partir de 2012 cambió radicalmente la situación y estas empresas quebraron, desaparecieron o fueron absorbidas (Matti, 2014). En el caso del área metropolitana de Valencia, el retroceso del sector está relacionado tanto con el cierre de la maraña de pequeñas empresas fundadas para aprovechar la oportunidad creada por el decreto, como con los procesos posteriores de concentración y desinversión por parte de las empresas multinacionales, desanimadas por el cambio normativo.

El segundo sector más afectado, especialmente en el ámbito nacional y local, es la construcción, cuyo número de empresas se ha reducido en un $-73,3 \%$. El retroceso de las empresas del sector contrasta con el incremento 
del número de UTE, especialmente las relacionadas con el ámbito local y nacional. Pese a la situación crítica de la construcción, el sistema de colaboración entre empresas que supone la UTE a la hora de concurrir a licitaciones públicas sigue siendo ventajoso, especialmente en un momento en que estas se enfrentan a graves dificultades de financiación.

Por el contrario, los sectores que han experimentado un menor retroceso son las manufacturas, especialmente las de mayor nivel tecnológico, y los servicios. Dado que estos son los sectores más vinculados al ámbito internacional (Salom, 2014), podemos concluir que la inserción en este tipo de redes parece haber sido un factor positivo para las empresas implicadas.

\subsection{Pautas territoriales}

Las relaciones financieras interempresariales de las empresas valencianas se establecen a distintas escalas territoriales, aunque predominan las escalas local y nacional. El $46 \%$ de las relaciones de control y el $17 \%$ de las relaciones de subsidiariedad recogidas por Orbis (2013) para el área funcional de Valencia se limitan al ámbito local. Un 34,6 \% y un 67,5\% adicionales se ejercían sobre el resto del territorio nacional, y solo un $19 \%$ de las relaciones de control y algo más del $15 \%$ de las relaciones de subsidiariedad afectaban al ámbito internacional. En este contexto, lo único destacable es el porcentaje relativamente elevado de relaciones de control de ámbito intercontinental (14\%), derivadas del establecimiento de filiales en países iberoamericanos por parte de las empresas valencianas (tabla 6).

La crisis económica ha tenido ciertos efectos sobre este patrón. Por un lado, el hundimiento de la construcción y las energías renovables han afectado las relaciones ejercidas en el ámbito local, que se han desplomado entre las dos fechas estudiadas, con un descenso del $-64 \%$. En segundo lugar, la crisis del sector de la construcción ha tenido también impacto en las relaciones, tanto de propiedad como de filiación, con empresas ubicadas en el resto de España (retrocesos del 24 y $12 \%$, respectivamente). Pero los aspectos más interesantes se refieren al ámbito internacional. Por un lado, en el ámbito europeo se ha

Tabla 6. Ámbito territorial de las relaciones de control (filiales cuya sede está localizada en el área urbana de Valencia) y de subsidiariedad (sedes cuya filial está localizada en el área urbana de Valencia) en 2013

\begin{tabular}{lrrrrrr}
\hline & \multicolumn{3}{c}{ De control } & & \multicolumn{2}{c}{ De subsidiariedad } \\
\cline { 2 - 3 } Tipo de enlaces & Número & $\%$ & & Número & $\%$ \\
\hline Intercontinentales & 47 & 14,03 & & 23 & 2,52 \\
Internacionales intracontinentales & 17 & 5,07 & & 118 & 12,94 \\
Intranacionales no intraurbanos & 116 & 34,63 & & 616 & 67,54 \\
Intraurbanos & 155 & 46,27 & & 155 & 17,00 \\
\hline Total & 335 & 100 & & 912 & 100 \\
\hline
\end{tabular}

Fuente: Orbis, Univ. de Lausana (2013), y elaboración propia. 
Tabla 7. Relaciones financieras por ámbito geográfico. Evolución 2010-2013 (\%)

\begin{tabular}{lcccccc}
\hline \multirow{2}{*}{\multicolumn{1}{c}{ Ámbito geográfico }} & \multicolumn{2}{c}{ Valencia } & & \multicolumn{2}{c}{ España } \\
\cline { 2 - 3 } \cline { 5 - 6 } \cline { 5 - 6 } & & Propiedad & Subsidiariedad & & Propiedad & Subsidiariedad \\
\hline Intercontinentales & 2,2 & 35,3 & & 7,1 & 30,2 \\
Internacionales intracontinentales & $-52,8$ & 18,0 & & $-4,3$ & 7,0 \\
Intranacionales no intraurbanos & $-23,7$ & $-12,0$ & & $-19,1$ & $-19,1$ \\
Intraurbanos & $-64,5$ & $-64,5$ & & - & - \\
\hline Total & $-50,1$ & $-27,3$ & & $-11,8$ & $-27,3$ \\
\hline
\end{tabular}

Fuente: Orbis.

Tabla 8. Localización geográfica de las sedes de empresas cuya filial está ubicada en el área funcional de Valencia. Se excluyen las localizadas en la propia área urbana

\begin{tabular}{|c|c|c|c|c|c|c|}
\hline \multirow[b]{2}{*}{ Continente y país de la filial } & \multicolumn{3}{|c|}{2010} & \multicolumn{3}{|c|}{2013} \\
\hline & Rango & $\begin{array}{l}\text { Número de } \\
\text { enlaces }\end{array}$ & $\%$ & Rango & $\begin{array}{l}\text { Número de } \\
\text { enlaces }\end{array}$ & $\%$ \\
\hline Total 10 primeros países & 1 a 10 & 211 & 90,2 & 1 a 10 & 159 & 88,3 \\
\hline Europa & & 188 & 80,3 & & 121 & 67,2 \\
\hline España & 1 & 152 & 65,0 & 1 & 116 & 64,4 \\
\hline Francia & 3 & 8 & 3,4 & 5 & 4 & 2,2 \\
\hline Reino Unido & 5 & 7 & 3,0 & - & 0 & 0,0 \\
\hline Portugal & 8 & 4 & 1,7 & 6 & 4 & 2,2 \\
\hline Suiza & 8 & 4 & 1,7 & - & 0 & 0,0 \\
\hline Mónaco & - & 0 & 0,0 & 8 & 3 & 1,7 \\
\hline Norteamérica & & 14 & 6,0 & & 11 & 6,1 \\
\hline Estados Unidos & 3 & 8 & 3,4 & 3 & 7 & 3,9 \\
\hline México & 7 & 6 & 2,6 & 10 & 3 & 1,7 \\
\hline Latinoamérica & & 25 & 10,7 & & 24 & 13,3 \\
\hline Brasil & 2 & 9 & 3,8 & 2 & 9 & 5,0 \\
\hline Argentina & 5 & 7 & 3,0 & 7 & 3 & 1,7 \\
\hline Chile & 10 & 3 & 1,3 & 11 & 2 & 1,1 \\
\hline Venezuela & 10 & 3 & 1,3 & 11 & 2 & 1,1 \\
\hline Colombia & - & 0 & 0,0 & 3 & 7 & 3,9 \\
\hline África & & 2 & 0,9 & & 5 & 2,8 \\
\hline Asia & & 4 & 1,7 & & 7 & 3,9 \\
\hline Oceanía & & 1 & 0,4 & & 0 & 0,0 \\
\hline Total & & 234 & 100,0 & & 180 & 100,0 \\
\hline
\end{tabular}

Fuente: BvD, Univ. de Lausana y elaboración propia.

producido un aumento de la dependencia, ya que el número de relaciones de participación de empresas europeas en las empresas valencianas ha crecido un $18 \%$, mientras que paralelamente se reducía la participación de empresas valencianas en empresas del resto de Europa en algo más de un $-50 \%$; por otra parte, se han incrementado notablemente las relaciones financieras, tanto de 
Tabla 9. Principales áreas urbanas con las que se mantienen relaciones de subsidiariedad. Los totales excluyen las relaciones locales

\begin{tabular}{lrrrrrr}
\hline & \multicolumn{2}{c}{2007} & & \multicolumn{2}{c}{2013} \\
\cline { 2 - 3 } \cline { 7 - 8 } \multicolumn{1}{c}{ Principales áreas funcionales urbanas } & & Enlaces & \multicolumn{1}{c}{$\%$} & & Enlaces & $\%$ \\
\hline España & 701 & 85,8 & & 609 & 80,4 \\
$\quad$ Madrid & 476 & 58,26 & & 447 & 59,05 \\
Barcelona & 121 & 14,81 & & 98 & 12,95 \\
Bilbao & 21 & 2,57 & & 20 & 2,64 \\
Sevilla & 21 & 2,57 & & 19 & 2,51 \\
\hline Otros países & 116 & 14,2 & & 141 & 18,6 \\
$\quad$ París & 18 & 2,2 & & 33 & 4,36 \\
$\quad$ Londres & 16 & 1,96 & & 10 & 1,32 \\
\hline Sin datos & - & - & & 7 & 0,9 \\
\hline Enlaces de filiación externos totales & 817 & 100 & & 757 & 100 \\
\hline
\end{tabular}

Fuente: BvD, Univ. de Lausana y elaboración propia.

Tabla 10. Localización geográfica de las filiales de empresas cuya sede está ubicada en el área funcional de Valencia. Los totales excluyen las localizadas en la propia área urbana

\begin{tabular}{|c|c|c|c|c|c|c|}
\hline \multirow[b]{2}{*}{$\begin{array}{l}\text { Continente y } \\
\text { país de la filial }\end{array}$} & \multicolumn{3}{|c|}{2010} & \multicolumn{3}{|c|}{2013} \\
\hline & Rango & $\begin{array}{c}\text { Número } \\
\text { de enlaces }\end{array}$ & $\%$ & Rango & $\begin{array}{c}\text { Número } \\
\text { de enlaces }\end{array}$ & $\%$ \\
\hline Total 10 primeros países & 1 a 10 & 211 & 90,17 & 1 a 10 & 159 & 88,33 \\
\hline Europa & & 188 & 80,34 & & 121 & 67,22 \\
\hline España & 1 & 152 & 64,96 & 1 & 116 & 64,44 \\
\hline Francia & 3 & 8 & 3,42 & 5 & 4 & 2,22 \\
\hline Reino Unido & 5 & 7 & 2,99 & - & 0 & 0,00 \\
\hline Portugal & 8 & 4 & 1,71 & 6 & 4 & 2,22 \\
\hline Suiza & 8 & 4 & 1,71 & - & 0 & 0,00 \\
\hline Mónaco & - & 0 & 0,00 & 8 & 3 & 1,67 \\
\hline Norteamérica & & 14 & 5,98 & & 11 & 6,11 \\
\hline Estados Unidos & 3 & 8 & 3,42 & 3 & 7 & 3,89 \\
\hline México & 7 & 6 & 2,56 & 10 & 3 & 1,67 \\
\hline Latinoamérica & & 25 & 10,68 & & 24 & 13,33 \\
\hline Brasil & 2 & 9 & 3,85 & 2 & 9 & 5 \\
\hline Argentina & 5 & 7 & 2,99 & 7 & 3 & 1,67 \\
\hline Chile & 10 & 3 & 1,28 & 11 & 2 & 1,11 \\
\hline Venezuela & 10 & 3 & 1,28 & 11 & 2 & 1,11 \\
\hline Colombia & - & 0 & 0,00 & 3 & 7 & 3,89 \\
\hline África & & 2 & 0,85 & & 5 & 2,78 \\
\hline Asia & & 4 & 1,71 & & 7 & 3,89 \\
\hline Oceanía & & 1 & 0,43 & & 0 & 0,00 \\
\hline Total enlaces & & 234 & 100 & & 180 & 100 \\
\hline
\end{tabular}

Fuente: BvD, Univ. de Lausana y elaboración propia. 
Figura 3. Localización de las sedes de las empresas filiales ubicadas en el AMV en 2013

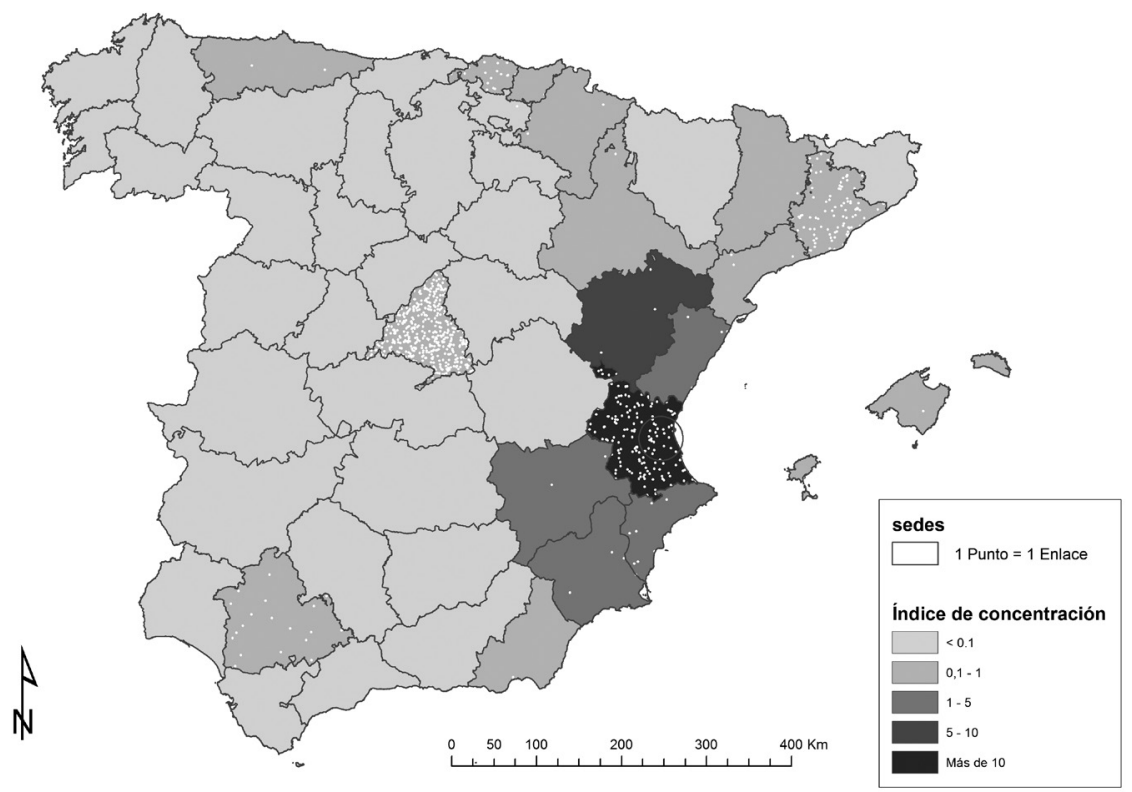

Nota: El índice de concentración relativa mide el peso relativo de las relaciones entre el área urbana funcional de Valencia y cada provincia en comparación con el peso de cada provincia en el conjunto de las relaciones de propiedad españolas. Un índice superior a 1 indica una relación preferencial de esa provincia con el área urbana de Valencia, mientras que un índice inferior a 1 indica lo contrario.

Fuente: BvD, Univ. de Lausana y elaboración propia.

propiedad como sobre todo de subsidiariedad, con los países iberoamericanos. Esta evolución es similar a la experimentada por el conjunto de España, aunque más acentuada (tabla 7).

A una escala más detallada, las relaciones de subsidiariedad en el ámbito internacional muestran un retroceso generalizado de las conexiones con el resto de países europeos, mientras que se mantienen a un nivel similar las existentes con los países iberoamericanos y Portugal. En general, existe una tendencia a la diversificación geográfica pese a que, en el ámbito de las relaciones interurbanas internacionales, París y Londres siguen siendo los centros urbanos más importantes. A escala nacional, se acentúa la pauta geográfica existente en el periodo previo a la crisis, con una cierta concentración relativa a lo largo del eje mediterráneo (figura 3), así como el predominio de la vinculación con las áreas funcionales de Madrid (que mantiene su primacía) y Barcelona.

Las relaciones de propiedad muestran también tendencias similares: un aumento de la diversificación geográfica, una reducción significativa de las relaciones con los países europeos, y un importante incremento de la vinculación con los países iberoamericanos, fruto de las estrategias empresariales de búsqueda de alianzas y mercados internacionales. A este respecto, resulta 
Tabla 11. Principales áreas urbanas con las que se mantienen relaciones de propiedad. Los totales excluyen las relaciones locales

\begin{tabular}{lrrrrrr}
\hline & \multicolumn{2}{c}{2007} & & \multicolumn{2}{c}{2013} \\
\cline { 7 - 7 } \cline { 6 - 7 } & Área funcional & Enlaces & $\%$ & & Enlaces & $\%$ \\
\hline España & 152 & 65,0 & & 116 & 64,4 \\
Madrid & 53 & 22,7 & & 44 & 24,4 \\
Barcelona & 11 & 4,7 & & 11 & 6,1 \\
Murcia & 8 & 3,4 & & 6 & 3,3 \\
Zaragoza & 3 & 1,3 & & 5 & 2,8 \\
Alicante & 6 & 2,6 & & 1 & 0,6 \\
\hline Otros países & 82 & 35,0 & & 64 & 35,6 \\
Lisboa & 1 & 0,4 & & 3 & 1,7 \\
Medellín & 0 & 0,0 & & 3 & 1,7 \\
París & 3 & 1,3 & & 1 & 0,6 \\
Londres & 5 & 2,1 & & 0 & 0,0 \\
\hline Enlaces de propiedad externos totales & 234 & 100,0 & & 180 & 100,0 \\
\hline
\end{tabular}

Fuente: BvD, Univ. de Lausana y elaboración propia.

Figura 4. Localización de las filiales de empresas ubicadas en el área metropolitana de Valencia en 2013

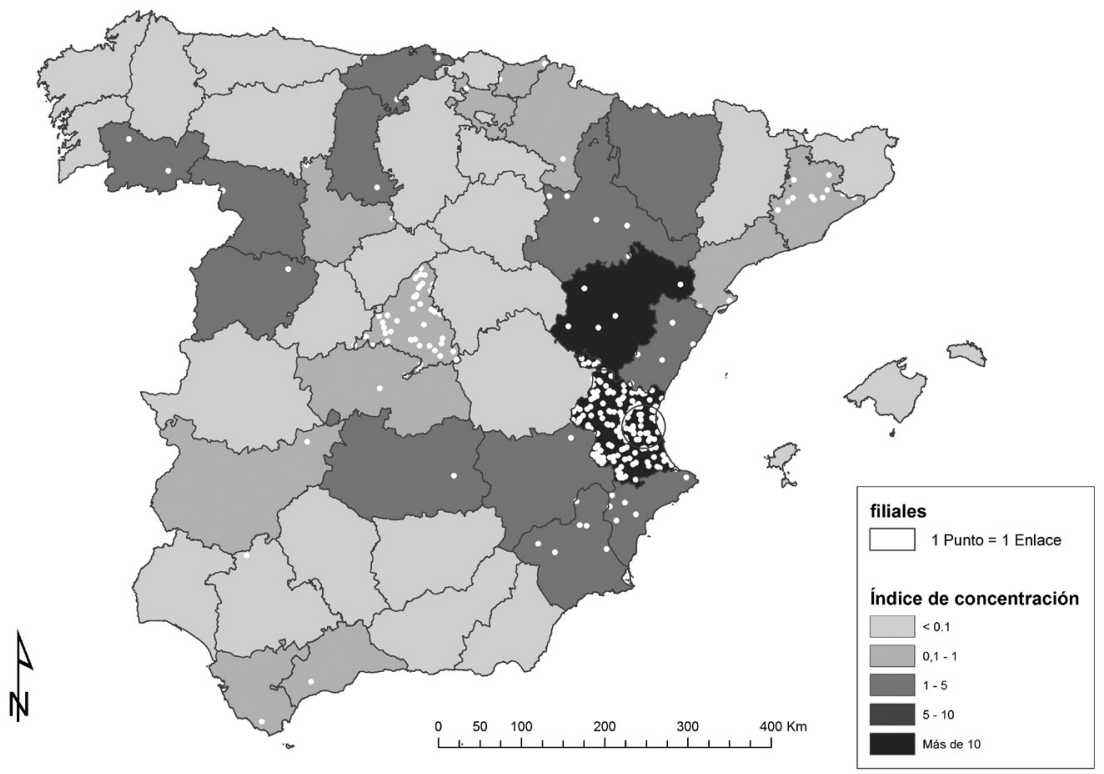

Nota: El índice muestra la concentración relativa de las relaciones de propiedad del área metropolitana de Valencia con cada provincia teniendo en cuenta el peso de dicha provincia en el conjunto de las relaciones de propiedad españolas. Un índice superior a 1 significa un número de relaciones con esa provincia superior al peso relativo de esa provincia en el total de España.

Fuente: BvD, Univ. de Lausana y elaboración propia. 
significativo que las relaciones con las áreas funcionales de Lisboa y Medellín, si bien aún escasas, pasen por delante de las tradicionales relaciones con París y Londres. A escala nacional, y pese a que el reducido número de enlaces dificulta la interpretación, cabe destacar la inexistencia de una pauta geográfica basada en la proximidad, así como la tendencia a concentrar las relaciones en las áreas metropolitanas de Madrid y Barcelona (tablas 10 y 11 y figura 4).

\section{Conclusiones}

La crisis económica ha tenido un fuerte impacto en el sistema de relaciones financieras de las empresas multinacionales valencianas. Caracterizada inicialmente como un núcleo de segundo nivel en el sistema global de ciudades, con una función principal de articulación en la red de relaciones de propiedadfiliación de las empresas multinacionales en el este y sureste de España, el Área Metropolitana de Valencia ha experimentado una significativa reducción de conectividad financiera, debido tanto a la desaparición de empresas por la crisis económica, más intensa que la media nacional, como a la simplificación de la red de relaciones en el caso de las energías renovables y de la construcción, afectadas por el estallido de sus burbujas respectivas. El efecto de la crisis ha supuesto también una mayor debilidad financiera de las empresas, que se ha traducido en un aumento de la dependencia financiera del área.

Sin embargo, la posición de las empresas en la red de relaciones y el ámbito geográfico con que se relacionan han sido factores de diferenciación relevantes. Ambas características están vinculadas a la especialización sectorial. Así, el ámbito más perjudicado ha sido el local, afectado por la crisis de las energías renovables y del sector de la construcción. En este ámbito es donde se ha producido la crisis más intensa, con un importante descenso del número de empresas y una significativa reestructuración financiera. Los efectos de la crisis han afectado tanto a las empresas intermediarias locales como, sobre todo, a sus empresas filiales localizadas en el área metropolitana de Valencia. También han experimentado un retroceso importante las conexiones en el ámbito nacional debido a la crisis del sector de la construcción, aunque la disminución del número de relaciones financieras ha sido menor en este ámbito que en el local, debido sobre todo al mantenimiento de la fórmula UTE como estrategia necesaria de las empresas para afrontar proyectos ante los problemas de financiación. Por el contrario, las relaciones financieras interempresariales establecidas en el ámbito internacional, con predominio de los sectores manufactureros de tecnología media y alta y de servicios intensivos en conocimiento, se han mantenido e incluso intensificado. Las empresas que mejor han resistido son las que funcionan como nodos intermedios en esta red de relaciones externas, que a la vez son filiales de sedes localizadas en el exterior y sedes de filiales ubicadas fuera del área metropolitana.

Finalmente, se ha producido un cambio en las pautas geográficas de las relaciones externas, producto de las estrategias de supervivencia de las empresas, que se ha concretado en la diversificación geográfica de sus redes financieras, especialmente hacia Iberoamérica y Portugal. Más específicamente, y en rela- 
ción con el ámbito internacional, ha aumentado el número de países implicados, sobre todo iberoamericanos (Brasil, Colombia, México, Argentina, Chile, Venezuela...), pero también otros como Portugal y Estados Unidos, frente a la reducción del peso de Francia y el Reino Unido. A escala nacional, el patrón geográfico es similar al existente en el periodo previo a la crisis; es decir, una cierta concentración relativa de las relaciones a lo largo del eje mediterráneo y un fuerte predominio de las conexiones con las áreas funcionales de Madrid (59\% de las relaciones de subsidiariedad externas y $29 \%$ de las de propiedad) y, a gran distancia, Barcelona (13\% y $6 \%$, respectivamente).

¿Qué significa esto en relación con la posición a escala global de la ciudad? En primer lugar, un claro descenso en la jerarquía, tanto a escala internacional como nacional. El hundimiento en particular del sector de la construcción, en el cual se había centrado una parte importante de los recursos financieros, y que permitía que Valencia se situara a escala nacional como un nodo de segundo nivel, tras Madrid y Barcelona, ha supuesto una pérdida de poder económico en el ámbito nacional. En segundo lugar, el retroceso de las participaciones externas en empresas locales certifica que se ha producido una pérdida del «atractivo» de la ciudad desde el punto de vista financiero. En tercer lugar, la relación entre "salidas» $\mathrm{y}$ «entradas», entre "poder» $\mathrm{y}$ "capacidad de atracción» ha derivado en un aumento de la dependencia financiera, $y$, por ende, en una pérdida de poder de decisión. Sin embargo, una parte de la red de relaciones, especialmente la referida a los sectores industriales de nivel tecnológico medio, se ha mantenido, en gran parte debido a una reorientación de sus estrategias a escala internacional que ha implicado su diversificación geográfica.

Por tanto, nuestras hipótesis iniciales en relación con los importantes efectos que ha tenido la crisis económica en el ámbito de las relaciones urbanas globales, así como el papel relevante de las empresas posicionadas en la red internacional, parecen confirmarse. No obstante, queda por determinar en qué medida la evolución que hemos descrito refleja una tendencia generalizada de las ciudades españolas, de lo experimentado por un determinado nivel de la jerarquía urbana, o si se trata de factores específicos del área metropolitana de Valencia que podemos achacar a una situación de peculiar debilidad derivada de la mayor intensidad con que han sido afectadas por la crisis las empresas valencianas. Para ello será necesario desarrollar estudios más extensos y sistemáticos que abarquen el conjunto de la jerarquía urbana española y permitan la comparación con otros ámbitos geográficos.

\section{Referencias bibliográficas}

Alderson, A. S. y BeCKField, J. (2004). «Power and Position in the World City». American Journal of Sociology, 109 (4) (enero), 811-851. $<$ http://dx.doi.org/10.1086/378930>

Allen, J. (2010). «Powerful City Networks: More than Connections, Less than Domination and Control». Urban Studies, 47 (13) (noviembre), 2.895-2911. $<$ http://dx.doi.org/10.1177/0042098010377364> 
Bellwald, A. y Rozenblat, C. (2012). «Pouvoirs et attractivités de l'aire urbaine de Paris dans les réseaux mondiaux d'entreprises multinationales». Note Rapide $I A U I d F$. Université de Lausanne e Institut d'Aménagement et d'Urbanisme de la région d'Île-de-France.

BRUNET, R. (1989). Les villes 'européennes'. París: RECLUS-DATAR.

CASTELls, M. (1996). The Rise of the Network Society. Malden, MA: Blackwell.

- (2010). "Globalisation, Networking, Urbanisation: Reflections on the Spatial Dynamics of the Information Age». Urban Studies, 47 (13), 2.737-2.745. <http://dx.doi.org/10.1177/0042098010377365>

Cattan, N.; Pumain, D.; Rozenblat, C.; Saint-Julien T. (1994). Le système des villes européennes. París: Anthropos, p. 201.

Cohen, R. B. (1981). "The new international division of labour, multinational corporations, and urban hierarchy». En: M. DEAR y A. J. SCOTT (eds.). Urbanization and Urban Planning in Capitalist Society. Nueva York: Methuen, 287-315.

Córdoba Ordóñez, J. A. y Gago García, C. (2010). «Latin American Cities and Globalisation: Change and Permanency in the Context of Development Expectations». Urban Studies, 47 (9). <http://dx.doi.org/10.1177/0042098010372680>

De MatTos, C. A. (2010). "Globalización y metamorfosis metropolitana en América Latina. De la ciudad a lo urbano generalizado». Revista de Geografía Norte Grande, 47, 81-104. <http://dx.doi.org/10.4067/S0718-34022010000300005>

DerudDer, B. y WiTlox, F. (2005). "An appraisal of the use of airline data in assessments of the world city network». Urban Studies, 42 (13), 2.371-2.388. <http://dx.doi.org/10.1080/00420980500379503>

- (2008). «Mapping global city networks through airline flows: context, relevance, and problems». Journal of Transport Geography, 16, 305-312. <http://dx.doi.org/10.1016/j.jtrangeo.2007.12.005>

FERIA, J. M. (2008). «Un ensayo metodológico de definición de las áreas metropolitanas en España a partir de la variable residencia-trabajo». Investigaciones Geográficas, 46, 49-68. <http://dx.doi.org/10.14198/INGEO2008.46.03>

Friedmann, J., y Wolff, G. (1982). "World City Formation: An Agenda for Research and Action». International Journal of Urban and Regional Research, 3, 309-344. <http://dx.doi.org/ 10.1111/j.1468-2427.1982.tb00384.x>

Gavinha, J. A. (2008). "Veinte años de ciudades globales: ideas, mitos y nuevas evidencias». Scripta Nova. Revista Electrónica de Geografía y Ciencias Sociales, vol. XII, núm. $270(6)$.

Halbert, L; Cicille, P.; Pumain, D. y Rozenblat, C. (2012). Quelles métropoles en Europe? Analyse comparée. Synthèse. París: DATAR. Travaux en Ligne, 11, 20 pp.

Hall, P. (1966). The World Cities. Londres, Weidenfeld and Nicolson.

KrÄTKE, S. (2014). "Global Pharmaceutical and Biotechnology Firms' Linkages in the World City». Urban Studies, 51 (6), 1.196-1.213. <http://dx.doi.org/10.1177/0042098013494420>

Luthi, S.; Thierstein, A. y Bentlage, M. (2013). «The Relational Geography of the Knowledge Economy in Germany: On Functional Urban Hierarchies and Localised Value Chain Systems». Urban Studies, 50 (2), 276-293. <http://dx.doi.org/10.1177/0042098012452325> 
MatTI, C. (2014). The Spanish wind energy rise. Pathways of knowledge creation within a multilevel environmental governance system. Valencia: Universidad de Valencia. Tesis doctoral.

Pflieger, G. y Rozenblat, C. (2010). «Introduction. Urban Networks and Network Theory: The City as the Connector of Connector of Multiple Networks». Urban Studies, 47 (13), 2.723-2.735. <http://dx.doi.org/10.1177/0042098010377368>

Rozenblat, C. (2004). Tissus de villes. Réseaux et systèmes urbains en Europe. Lausana: Université de Lausanne. Tesis doctoral.

- (2010). "Opening the Black Box of Agglomeration Economies for Measuring Cities. Competitiveness through International Firm Networks». Urban Studies, 47 (13), 2.841-2.865. <http://dx.doi.org/10.1177/0042098010377369>

Rozenblat, C. y Pumain, D. (2007). «Firm linkages, innovation and the evolution of urban systems». En: TAYLOR et al. (eds.). Cities in Globalization: Practices, policies and theories. Londres: Routdledge, 130-56.

Rozenblat, C.; Zaidi, F. y Bellwald, A. (2016). "The multipolar regionalization of cities in multinational firms' networks». Global Networks. <http://dx.doi.org/10.1111/glob.12130>

SALOM, J. (2011). «Procesos territoriales y transformaciones recientes del sistema urbano valenciano». Scripta Nova. Revista Electrónica de Geografía y Ciencias Sociales, XV (356).

- (2014). «La inserción de las ciudades en las redes globales a través de las relaciones financieras. El caso de la ciudad de Valencia». Cuadernos de Geografía, 95/96, 101123.

SASSEn, S. (1991). The Global City. Princeton: Princeton University Press. <http://dx.doi.org/10.1177/027046769301300240>

Smith, R. G. (2014). «Beyond the Global City Concept and the Myth of 'Command and Control'». International Journal of Urban and Regional Research, 38 (1), 98-115. <http://dx.doi.org/10.1111/1468-2427.12024>

TAYLOR, P. J. (2004). World City Network: A Global Urban Analysis. Londres: Routledge.

Wall, R. S. y VAn der KnaAp, G. A. (2011). «Sectoral Differentiation and Network Structure Within Contemporary Worldwide Corporate Networks». Economic Geography, 87 (3), 267-308.

<http://dx.doi.org/10.1111/j.1944-8287.2011.01122.x> 